\title{
"El artificio de los alquimistas". \\ Notas sobre La gaya ciencia, de Nietzsche
}

\section{"The Artifice of the Alchemists" \\ Notes on The Gay Science, by Nietzsche}

Antonio Castilla Cerezo

Universidad de Barcelona

\section{Resumen}

Asumiendo, por un lado, que como dijo Giorgio Colli La gaya ciencia es el momento central de la vida de Nietzsche y, por otro, que en ese libro el filósofo alemán es particularmente cuidadoso con la elección de los títulos, este artículo se propone analizar la relación existente en dicha obra entre los términos que vincula implícitamente el título de la misma, a saber: la ciencia y la alegría. Para ello recurre, además de a ciertos momentos estratégicos del texto publicado por Nietzsche, a diversos pasajes extraídos de su correspondencia y de sus escritos póstumos, así como a las aportaciones de pensadores posteriores (tales como Michel Foucault o el mismo Giorgio Colli) que reflexionaron sobre la mencionada conexión.

Palabras clave: Nietzsche, ciencia, alegría, Foucault, Colli.

\section{Abstract}

Assuming, on the one hand, that as said Giorgio Colli The Gay Science is the central moment of Nietzsche's life and, secondly, that in this book the German philosopher is particularly careful 
with the choice of titles, this article analyzes the relationship in that work between the terms that implicitly links the title of it, namely science and joy. For this purpose uses, in addition to certain strategic points in the text published by Nietzsche, various passages from his correspondence and his posthumous writings, as well as the contributions of later thinkers (such as Michel Foucault or the same Giorgio Colli) that reflected on said connection.

Keywords: Nietzsche, Science, Joy, Foucault, Colli.

\section{La alquimia como arte}

T a primera edición de La gaya ciencia, publicada en julio de 1882, contenía un total de cuatro libros, precedidos por sesenta y tres poemas breves (reunidos bajo el título «Burla, astucia y venganza. Preludio en rimas alemanas»). ${ }^{1}$ No fue hasta la segunda edición, publicada cinco años más tarde, que Nietzsche añadió un quinto libro, un prefacio y las «Canciones del Príncipe Vogelfrei», dando así al texto su forma definitiva. La primera edición de esta obra acababa, por lo tanto, con el Libro cuarto, titulado «Sanctus Januarius» en alusión al mes de enero de 1882 ("el más prodigioso mes de enero", según Nietzsche), que concluye (si descontamos el breve «Epílogo» final, reproducido literalmente en Así habló $\mathrm{Za}$ ratustra) con el célebre aforismo 341, donde el filósofo hace referencia por primera vez a la gran revelación del eterno retorno, que había tenido durante el verano del año anterior en Sils Maria.

Este dato, en principio de un interés meramente biobibliográfico, ha de ser entendido según Germán Cano como un síntoma de que el «Sanctus Januarius» era para Nietzsche algo más que el cuarto libro de La gaya ciencia. No es, sin embargo, ni mucho

1 En lo relativo a la traducción de títulos y fragmentos de La gaya ciencia, cito a partir de la traducción de esta obra incluida en Nietzsche, 2014. 
menos el único signo visible que nos invita a pensar de este modo: en ese mismo sentido apuntan, por ejemplo, las cartas que nuestro autor dirigió a sus amigos para preguntarles qué impresión les había producido este cuarto libro, dejando de lado los tres anteriores; pero también ( $y$, como veremos, en algún sentido sobre todo) un fragmento póstumo (M III 6b. Primavera de 1882, 19[12]) (Nietzsche, 2008: 906) por el que sabemos que consideró la posibilidad de colocar este cuarto libro al comienzo de la obra, como su primera parte. En un texto extremo como éste, que sólo acepta ser colocado al principio o al final de la obra a la que pertenece, es donde pienso que debemos buscar la pista que nos permita adentrarnos en la lectura de la misma. Pero entonces, ¿acaso no tendríamos que preguntarnos, antes que nada, cuál es la pretensión de aquel cuarto libro, con el que parece razonable pensar que mantienen algún tipo de vínculo las múltiples líneas problemáticas que atraviesan, en un itinerario no siempre evidente para el lector, el resto de La gaya ciencia?

Para intentar responder a esta interrogante, sigo de nuevo a Germán Cano, autor según el cual Nietzsche quiso ofrecer en el "Sanctus Januarius» "una máscara nueva, una especie transmutación alquímica ligada a una nueva experiencia gozosa de la subjetividad" (Cano, 2001: 19). Esta alusión a la alquimia me hace pensar, como mínimo, en el siguiente par de textos: en primer lugar, en una carta dirigida a Franz Overbeck el 25 de diciembre de 1882, en la que Nietzsche escribe que, si no consigue inventar "el artificio de los alquimistas para transformar todo este fango en oro" (Nietzsche, 2010: 306), está perdido, añadiendo inmediatamente que ahora tiene la más bella ocasión para demostrar que todos los acontecimientos le son útiles, todos los días santos y todos los hombres divinos -lo que nos permite hacernos una idea bastante aproximada de lo que entendía este filósofo por "alquimia"; en segundo lugar, en el aforismo 292 de La gaya ciencia, donde se acusa 
a los moralistas de dominar "el arte inverso al de la alquimia, la desvalorización de lo más valioso" (Nietzsche, 2014: 836). Esta caracterización de la alquimia como arte no parece en absoluto casual -no olvidemos que, según Nietzsche, lo más contrario al arte es la moral y la corrupción del arte no se puede llevar hasta sus últimas consecuencias si no es desde una moral determinada. Es por esto que la prolongación lógica de dicho aforismo se encuentra un poco más adelante, en el 299, donde se afirma explícitamente que lo que hemos de aprender del artista es "a hacer las cosas bellas, atractivas, deseables, cuando no lo son”, asunto decisivo para nuestro filósofo porque, como añade poco después, "en sí no lo son jamás" (Nietzsche, 2014: 839).

\section{Una ilusión retrospectiva}

Existen, obviamente, muchos tipos de formas artísticas, pero no todas son reconocidas como tales: tal es el caso, sobre todo, de los nombres. En La gaya ciencia Nietzsche no esconde su peculiar interés por los nombres, en los que piensa que se puede encontrar la condición para que la mayoría pueda llegar a ver las cosas (aforismo 261), y el conocimiento de los cuales llega a poner por encima del de las esencias (aforismo 58). Ahora bien, ¿cómo hemos de entender estas extrañas declaraciones en el seno de la obra a la que pertenecen? ¿Cuál es su función dentro de ésta?

Hay un dicho -creo que japonés- según el cual, para una persona que desconoce el Zen, las montañas son montañas y los ríos son ríos; cuando ha comenzado a conocerlo, las montañas dejan de ser montañas y los ríos ya no son ríos; finalmente, después de la iluminación, las montañas vuelven a ser montañas y los ríos vuelven a ser ríos. El aforismo 15 de La gaya ciencia utiliza la siguiente metáfora, que puede ser entendida como complementaria de la anterior: la montańa que, contemplada desde cualquier punto de 
vista, llena de encanto la comarca que domina resulta, una vez nos acercamos a ella y escalamos hasta su cima, decepcionante. Esta imagen sirve a Nietzsche para mostrarnos de una manera intuitiva que el conocimiento (y, particularmente, el conocimiento de nosotros mismos) no es algo recomendable para todo el mundo, sino sólo para aquellos que son capaces de soportar la verdad, prescindiendo de los velos protectores que proporciona la ignorancia. Pero esta misma imagen también nos puede servir para prevenirnos contra otra idea, según la cual sólo nos haría falta volver a alejarnos de la montańa para percibirla de nuevo en su unidad -pues lo cierto es que este alejamiento no nos garantiza por sí solo la recuperación de aquel primer efecto. Es preciso que hayamos sido capaces de soportar la prueba, la durísima prueba del conocimiento, para recuperar la percepción unitaria de la montańa, que sólo entonces seremos capaces de apreciar por el hecho mismo de haberla perdido (y vuelto a recuperar). En este instante privilegiado, la montańa vuelve a ser montaña, el río vuelve a ser río e incluso nosotros volvemos a ser nosotros mismos -en un proceso que culmina con la recuperación de una relación no problemática con nuestro propio nombre.

Pues bien, si nos fijamos ahora en el nombre (es decir, en el título) de La gaya ciencia, posiblemente adivinaremos en qué consiste una parte importante de su aventura. ¿Qué quiere decir esta expresión: «gaya ciencia»? Hasta el momento en que Nietzsche escribió este libro, la ciencia había sido considerada únicamente como algo serio y profundo, es decir, como un ámbito de estudios del cual la alegría y la superficialidad quedaban excluidas o, como mucho, eran integradas en el listado de los posibles objetos de estudio científico - con lo que la concepción de la ciencia como "cosa seria" no resultaba modificada en absoluto. Nietzsche no se confunde cuando identifica ciertas afirmaciones de Spinoza como la culminación de esta manera de entender la ciencia, que es justamente la que él 
quiere superar. Como tal vez recuerde el lector, en la Parte III de su Ética Spinoza define, de un lado, la alegría como "la pasión por la que el alma pasa a una perfección mayor" (proposición 11, escolio [a.]) (Spinoza, 2000: 134) o como "el paso del hombre de una perfección menor a una mayor" ("Definiciones de los afectos", ${ }^{2}$ ) (Spinoza, 2000: 170); pero, de otro, mantiene (proposición 58) que, además de esta alegría pasiva, existe una alegría que es acción. Para este filósofo (véase la proposición 15 de esa misma tercera parte) tanto la alegría como la tristeza pueden ser provocadas en nosotros por cualquier cosa, pero el tránsito desde la pasividad hasta la actividad (que no expondré aquí para no desviarme excesivamente del principal asunto que me ocupa) sólo es posible por la alegría, que en este punto se revela superior a la tristeza y, en consecuencia, como el más importante de todos los afectos. Podemos concluir, por lo tanto, que el conocimiento al que aspira Spinoza en Ética hace de la alegría uno de sus objetos más eminentes, sin que eso modifique la esencia misma de la ciencia, es decir, su carácter intrínsecamente ajeno a toda afectividad.

Es contra esta posición epistemológica que Nietzsche dirige una de sus más lúcidas invectivas, que se encuentra en el aforismo 333: "Non ridere, non lugere, neque detestare, sed intelligere!, dice Spinoza”; ${ }^{2}$ ahora bien, para que el conocimiento (que está íntimamente ligado a este intelligere, si es que no es idéntico a él) sea posible, cada uno de estos instintos ha de aportar "su opinión unilateral sobre la cosa o suceso" (Nietzsche, 2014: 850); a continuación surge "la lucha entre esas posiciones unilaterales y de ella, a veces, [...] una especie de justicia y de contrato" (Nietzsche, 2014: 850-851), gracias al cual "todos esos impulsos pueden afirmarse en la existencia y tener razón en común”. Son estas últimas escenas de reconciliación las que tomamos equivocadamente como punto de partida del conocimiento cuando pensamos "que intelligere es algo

2 En el I, 4 de su Tratado politico. 
conciliador, justo, bueno, algo esencialmente opuesto a los impulsos; mientras que sólo es una cierta relación de los impulsos entre sï (Nietzsche, 2014: 851). Parece como si de estas razones Nietzsche extrajera la siguiente consecuencia: si el intelligere no es extraño al ridere, al lugere y al detestare, si el conocimiento no se encuentra esencialmente desligado de los afectos (como tiende a hacernos creer la ilusión retrospectiva que aquí se ha intentado denunciar), sino que resulta del equilibrio de éstos, entonces el conocimiento tendrá que tener un vínculo privilegiado con aquel afecto que, según Spinoza, es el más importante de todos, es decir, con la alegría. Habrá que concebir entonces la profundidad científica como indisociable de la alegre superficialidad, en lugar de entender estos dos términos como opuestos irreconciliables -este es finalmente el lema de la vía que Nietzsche inaugura y explora bajo el título de La gaya ciencia.

\section{Un corazón roto}

Para recorrer esta vía (que tiene, ciertamente, algo de escolástico, pero de un escolasticismo asumido que, por ello, se asume como un juego posible entre otros y no como una imposición) podemos comenzar o bien partiendo de la ciencia o bien desde la alegría. Seguramente Nietzsche habría querido que escogiéramos indistintamente una cualquiera de estas dos opciones, pero lo cierto es que, si atendemos ahora nuevamente al título que escogió para esta obra, no nos será difícil darnos cuenta de que en él la ciencia sigue siendo lo sustantivo, mientras que la alegría no aparece en realidad sino como adjetivo, es decir, como lo "alegre". Optaré, en consecuencia, por el primero de aquellos dos posibles puntos de partida, no sin adoptar para ello determinadas precauciones; asunto éste en relación al cual me parece de utilidad recordar algunas de las observaciones que Foucault realizó durante la primera de las 
conferencias que pronunció entre el 21 y el 25 de mayo de 1973 en Rio de Janeiro, dentro de un ciclo organizado por la Pontificia Universidad Católica de esta ciudad, y que posteriormente han sido transcritas y publicadas bajo el título genérico de La verdady las formas jurídicas. En esta primera sesión, poco antes de señalar la importancia del mencionado aforismo 333, este filósofo francés hizo referencia a otro fragmento de La gaya ciencia, concretamente el 109, en el que Nietzsche afirmaba que no se ha de "presuponer en general y en todas partes algo tan pleno de forma como el movimiento cíclico de nuestras estrellas vecinas”, y que el carácter general del mundo es, al contrario, "para toda la eternidad, caos, no en el sentido de que falte la necesidad, sino en el de que falta el orden, la articulación, la forma, la belleza y como quieran llamarse todas nuestras estéticas categorías humanas" (Nietzsche, 2014: 794).

El verdadero punto de partida de esta reflexión se encuentra, sin embargo, en otro texto de Nietzsche, esta vez datado en 1873 y publicado póstumamente, al cual alude igualmente Foucault. Se trata de las primeras líneas de Sobre verdad y mentira en sentido extramoral, en las cuales se afirma que el conocimiento fue inventado una vez en una rincón perdido del universo, es decir, en un momento y en un lugar determinados. Según el autor de Vigilar $y$ castigar, el hecho de que el conocimiento sea entendido como una invención tiene implicaciones que no debemos pasar por alto. De entrada, cuando Nietzsche habla de "invención" (Erfindung) lo hace para oponer a la palabra "origen" (Ursprung), con lo que quiere decirnos que el conocimiento no está dado (como lo están ya las cosas en algún sentido en su origen) de una manera implícita, envuelto en la Naturaleza o en el Universo, y que por lo tanto no ha surgido de ninguna de estas dos instancias con una gran continuidad, sino que existe sólo como resultado de una ruptura, de modo que sólo resulta posible entender en qué cosiste "en esas relaciones de lucha y poder, en la manera como se odian entre sí 
los hombres, luchan, procuran dominarse unos a otros, quieren ejercer relaciones de poder unos sobre otros" (Foucault, 2000: 28). Considerada desde este punto de vista, La gaya ciencia no es sólo el texto donde se supera la posición cognoscitiva que aún presuponía Spinoza, sino también el escrito en el que Nietzsche parece decidido por primera vez a extraer las consecuencias de una obra de juventud que él nunca vio publicada, pero el latido de la cual se siente en un buen número de puntos de este libro, como si fuera su corazón secreto.

\section{La ciencia y el fenómeno}

Foucault nos recuerda también, en la misma conferencia, que el año en que Nietzsche escribió Sobre verdad y mentira... la academia alemana se encontraba en plena eclosión del neokantismo y que, según esta corriente filosófica (como para el propio Kant), espacio y tiempo son formas a priori del conocimiento. Esto quiere decir que para un neokantiano el tiempo y el espacio son condiciones que el sujeto impone a la realidad, de manera que no preexisten al conocimiento ni pueden, como quería el joven Nietzsche, ser algo parecido a las rocas primordiales sobre las cuales el conocimiento viene a fijarse. No por ello hemos de entender que la posición de Nietzsche no tenga ningún vínculo con la kantiana; al contrario, parece bastante evidente que la revaloración de las apariencias que encontramos en La gaya ciencia es una radicalización de la distinción fundamental del filósofo de Königsberg, o sea, de la que éste estableció entre la cosa en sí y el fenómeno. Si en la Crítica de la razón pura Kant nos decía que de la cosa en sí no podemos tener ningún conocimiento y que por ello el objeto de la ciencia es necesariamente el fenómeno, Nietzsche llegará aún más lejos al declarar (en el aforismo 354) que no le interesa en absoluto la contraposición entre estos dos términos, "porque estamos bien lejos 
de «conocer» lo suficiente como para poder siquiera diferenciar de este modo" y sólo “"sabemos» (o creemos o nos imaginamos) exactamente tanto como puede ser útil en interés del rebaño humano, de la especie" (Nietzsche, 2014: 869).

Este razonamiento es, sin embargo, completamente externo a los presupuestos del neokantismo y, por eso, un representante de este movimiento probablemente ni siquiera se inmutaría al escucharlo. Otra cosa habría sido si Nietzsche hubiera utilizado las mismas herramientas conceptuales que los neokantianos: entonces tal vez habría dicho que el problema de fondo de la distinción entre cosa en sí y fenómeno consiste en que, en virtud de las premisas kantianas, el procedimiento científico ha de continuar al margen de la alegría y de la superficialidad. En otras palabras, si como dice Kant (y repite el neokantismo), somos nosotros los que, en tanto que sujetos de conocimiento, imponemos las condiciones de la posibilidad del fenómeno, entonces no tiene sentido siquiera decir que nuestro conocimiento es alegre o triste, porque eso sería tanto como afirmar que el fenómeno afecta a las condiciones que nosotros le imponemos, con lo que... ¡nos afectaríamos a nosotros mismos! Pero ¿̇no es esta autoafección, esta autotransformación (que comporta sólo de manera subsidiaria una cierta dosis de autoconocimiento) el objeto último de aquel arte de la alquimia que, como hemos visto, Nietzsche consideró imprescindible para sí mismo? Llevar hasta este extremo la teoría kantiana de la relación entre la ciencia y el fenómeno me parece, junto con la extracción de las consecuencias de determinadas ideas de Sobre verdad y mentira... y la superación de la posición epistemológica que aún se encontraba en la Ética de Spinoza, una de las tres operaciones indispensables para entender cuál es la posición adoptada en La gaya ciencia en relación al conocimiento. 


\section{5. "Prolongar la danza terrenal"}

Una vez hechas estas consideraciones sobre la ciencia, es preciso que me dirija hacia aquello que constituye mi verdadero objetivo en estas páginas: el análisis de la relación entre aquélla y la alegría o, lo que es igual, entre la profundidad y las superficies. Sin duda, este problema atraviesa también otros escritos de Nietzsche, por no decir la totalidad de la obra de nuestro autor. ¿En qué se distingue a este respecto La gaya ciencia? Otro lector atento de este libro, Giorgio Colli, lo ha señalado como "«central» en la vida de Nietzsche” (Colli, 2000: 111), no sólo porque ocupa una posición intermedia dentro de su producción literaria, sino porque supone, además, por relación a los escritos que lo preceden y a aquellos que le seguirán, un momento mágico de equilibrio, la única experiencia de "salud" total de su autor. Esto se traduce en el hecho de que, más aquí que en Aurora, por citar el título inmediatamente anterior, y no digamos ya que en Así habló Zaratustra, que es su inmediato sucesor, encontramos en La gaya ciencia una tendencia a reabsorber las estridencias, a suprimir la animosidad y el fanatismo que acaso asciende -ella también- a la superficie en estado químicamente puro en el aforismo 324, significativamente titulado «In media vita».

Colli escoge, a pesar de esto, como ejemplo de esta operación (de la cual resulta un insospechado equilibrio que, según hemos visto, cuando se establece entre los instintos permite "inventar" el conocimiento, y la importancia del cual es muy superior en este libro que en ningún otro escrito de Nietzsche) el aforismo 54, donde su autor nos dice que ha despertado, pero sólo para entender que es preciso que continúe soñando para no sucumbir, como el sonámbulo necesita continuar sońando para no caer. A raíz de esto, la apariencia ya no es para él lo opuesto a un ser cualquiera, dado que de un ser no se pueden decir sino los atributos de su apa- 
riencia. De esto deduce Nietzsche que la apariencia no ha de ser minusvalorada por quien aspire a conocer, pues también "el hombre de conocimiento es un medio para prolongar la danza terrenal" (Nietzsche, 2014: 766). Según Colli, en este aforismo se neutralizan dos tendencias antitéticas presentes en la obra nietzscheana (de un lado, aquella que se establece entre la crítica al concepto metafísico de "apariencia” -en virtud de la cual éste sería lo opuesto a la "realidad"- y, de otro, el desarrollo de una concepción propia del mundo como mentira, es decir, de algo muy similar a la apariencia), dando como resultado una visión superior, contemplativa y más clara. Pero esta cima no podría ser alcanzada sin abandonar el lenguaje moral, para lo cual sería preciso, no sólo que la verdad y la mentira pudieran ser entendidas en sentido extramoral, sino que incluso el bien y el mal fueran concebidos de un modo completamente diferente al que hasta entonces los había considerado la moral. Véase en este sentido el aforismo 1, según el cual no existe distinción nítida entre estos dos términos desde el punto de vista de la utilidad de la especie (la cual, desde la perspectiva que aquí adopta Nietzsche, es lo más profundo que existe).

\section{El modelo arbóreo}

Para desplegar las consecuencias de este nuevo punto de vista, tal vez sea conveniente volver al fragmento póstumo ya citado, en el que se asigna a cada uno de los otros tres libros incluidos en la primera edición de La gaya ciencia un título (suprimido finalmente en esta edición) que nos permite hacernos una idea de los temas que constituyen sus núcleos respectivos. Según este plan inicial, después del «Sanctus Januarius» debía figurar un segundo libro llamado "Sobre artistas y mujeres», a continuación un tercero titulado "Pensamientos de alguien sin Dios" y, finalmente, un cuarto libro, "Del «diario moral»". Si hemos de creer el que sobre este 
punto nos dice Mazzino Montinari (Montinari, 199: 105-106), estos tres últimos bloques temáticos habrían conservado (con alguna modificación de detalle) su centralidad en aquella primera edición, en la cual no obstante se habría modificado su orden: así, el primer libro de esta edición indagaría el significado del «mal» para la historia de la humanidad (asunto que será reservado para el Libro cuarto en la ordenación que figura en el fragmento póstumo al que he aludido), mientras que el segundo abordaría tres cuestiones (el arte, la mujer -estas dos primeras, retomadas en Más allá del bien y del mal- y el mundo griego) directamente relacionadas con la necesidad de belleza (tema del Libro segundo también en la ordenación inicial de la obra) y, finalmente, el tercero se ocuparía de la supresión de todas aquellas "sombras divinas" que todavía se proyectan sobre nuestros valores culturales (tema del Libro tercero en aquella misma ordenación).

No intentaré aquí dar cuenta de las tesis principales que en relación a estos tres bloques temáticos se despliegan en La gaya ciencia, lo que me llevaría a sobrepasar ampliamente las dimensiones de un escrito como éste; en lugar de eso, quisiera solamente señalar la presencia de cierto tipo de operación que me ha parecido percibir en buena parte de lo que Nietzsche ha escrito sobre estos tres asuntos en la obra que me ocupa. Tomaré para ello como ejemplo lo que en ésta se dice a propósito de los artistas, no porque lo que Nietzsche escribió sobre los otros temas revista una importancia menor, sino porque durante la lectura de los aforismos que se refieren a aquéllos es probablemente donde más nos exponemos al riesgo de confundirnos. Esto ocurre porque Nietzsche, en este escrito como en otros, parece hablar unas veces en el lenguaje de todo el mundo y otras veces en un lenguaje propio, en el que los mismos términos utilizados por el lenguaje anterior tienen un significado distinto. Así, por ejemplo, en el aforismo 87 reprocha a los artistas el que valoren de una forma superficial sus mejores productos, con 
lo que se entiende implícitamente la superficialidad como algo negativo; en cambio, según el aforismo 256 lo que más aprecian de las cosas todos los hombres de la profundidad "es que tienen una superficie: su epidermicidad” (Nietzsche, 2014: 826). Este tipo de antinomias sólo pueden disolverse si entendemos que se está atribuyendo a una misma palabra un significado distinto en cada caso: habrá que distinguir, pues, entre el modo en que se instala en la superficie aquel que, por habitar siempre en ella, no se percata ya de la existencia de las profundidades y otro modo, el de quien tiene experiencia de la profundidad, para quien el acceso a la superficie (más aún, incluso la existencia de las superficies mismas) supone una experiencia totalmente distinta de la del primero. Rescatando una célebre distinción terminológica, podríamos decir incluso que este último tiene propiamente la experiencia de las superficies, en tanto que el primero sólo accede a la vivencia de las mismas. La contradicción queda resuelta, pues, en la medida en que en los dos aforismos, contra lo que pudiera parecer a primera vista, no se está hablando de lo mismo.

Pero no dejamos atrás una aporía de este estilo en La gaya ciencia sin que esa dificultad continúe persiguiéndonos bajo otras máscaras. Y es que podríamos pensar, en función de lo hasta aquí dicho, que Nietzsche se anticipa en este punto al modo en que Deleuze habló de las superficies en el siglo XX -es decir, reivindicando en primer lugar que lo más profundo es la piel (Valèry), para afirmar a continuación que la raíz más profunda es el rizoma, esto es, aquella que se halla más cerca de la superficie, extendiéndose horizontalmente para permanecer en las proximidades de ésta-. Nada de eso ocurre en Nietzsche, para quien el modelo desde el que se piensa la raíz (y, por consiguiente, la profundidad) sigue siendo el arbóreo, y no el rizomático. Esto es particularmente claro en el aforismo 371 , donde leemos que los hombres incomprensibles (entre los que el propio Nietzsche se incluye) hunden sus raíces "con cada 
vez más fuerza [...] en la profundidad -en el mal-”, mientras que al mismo tiempo abrazan "el cielo con un amor y una amplitud cada vez mayor", y absorben su luz cada vez más sediento con todas sus ramas y sus hojas, esto es, que crecen "como árboles [...], no en un solo sitio, sino en todas partes, no en una dirección sino tanto hacia arriba, hacia afuera, como hacia adentro y hacia abajo" (Nietzsche, 2014: 886).

Dejando de lado la equiparación que aquí se introduce de manera casi tangencial entre la profundidad y lo perverso (y que podría llevarnos a concluir que hay todavía en este aforismo un rastro de lenguaje moral), resulta innegable que Nietzsche conserva el modelo arbóreo, que ya había utilizado Descartes (en la célebre carta que dirigió al traductor de Los principios de la filosofía, y que hoy se utiliza habitualmente como prólogo a este libro) para caracterizar a la unidad conformada por la totalidad de las ciencias. Cierto que en La gaya ciencia, a diferencia de lo que sucedía en Descartes, la parte más importante del árbol de las ciencias es el tronco, es decir, la física, y no las raíces, o sea, la metafísica (véanse las últimas líneas del aforismo 335, en conjunción con las del 344), y que es como resultado de esta operación que Nietzsche cree que puede pensarse prescindiendo de la idea (metafísica) de Dios. Pero si esta interpretación es correcta, ¿cómo es entonces que en el aforismo 2 Nietzsche coincide con Descartes al reivindicar la demanda de la certeza (que es justamente lo que lleva a este último pensador a fundamentar la física sobre la metafísica) como el más íntimo de los deseos que experimentan aquellos hombres dotados de conciencia intelectual? ¿Y, suponiendo que esto último no constituya un verdadero problema para Nietzsche, cómo es que hacia el final del aforismo 347 éste afirma que el espíritu libre por excelencia será aquel que "se despida de toda creencia, de todo deseo de certeza”? (Nietzsche, 2014: 865) ¿Hay que pensar que, llegados a este punto, existen también dos sentidos para el término "certeza”, 
de tal manera que el último de ellos tendría que ver solamente con las profundidades -y sería, en consecuencia, rechazado por nuestro autor-, en tanto que el otro (empujado por la alegría, por la fuerza de la soberanía individual, por la libertad en el querer) sería a la vez abismo y danza, profundidad y altivez, conocimiento y alegría? Y, aun suponiendo que así sea, ¿̇nos garantiza esta maniobra la resolución de las dificultades que hemos planteado, esto es, la detención en este punto de las cadenas de aporías? ¿ O fue acaso la intención de Nietzsche prolongar esas cadenas, sumergirnos en un juego de espejos en el que de un problema saltamos a otro, así una y otra vez, sin remisión última a raíz alguna que pueda ser considerada la más profunda de todas? ¿Fue ese su modo de anticipar la concepción de la superficie como rizoma? ¿ $\mathrm{O}$, por el contrario, es sólo el signo visible de la persistencia del fanatismo, de los desequilibrios que en esta obra parecen abolidos, reequilibrados por la magia de una perspectiva superior más que en ningún otro escrito de su autor? Sin descartar, por supuesto, que se trate de ambas cosas al mismo tiempo.

\section{En qué medida somos todavía piadosos}

Nos falta, sin embargo, por esclarecer una de las cuestiones fundamentales de este texto. Y es que si, como he argumentado, el Libro cuarto nos da la clave para entender a qué se refiere Nietzsche cuando habla de "ciencia" en esta obra, en tanto que los tres primeros complementan la interpretación del título por el lado de la alegría -con la metáfora arbórea como imagen del tipo de unidad que conforman ambos aspectos al ser tomados conjuntamente-, entonces ¿qué es exactamente lo que aporta el Libro quinto? ¿No será acaso un mero revestimiento, una piel superpuesta a los cuatro libros anteriores -lo que, por descontado, no podría tener que ver más profundamente con lo expuesto en ellos? De ser así, entonces 
no tendría que sorprendernos en absoluto el que en este quinto libro se tratasen los mismos asuntos que en los cuatro anteriores, sólo que puestos, por así decir, en práctica, como si el árbol aludido previamente no fuera la conjunción de una raíz y una parte visible, sino un ser efectivamente vivo. Ahora bien, los seres vivos producen cosas (las hojas del árbol, por seguir con el mismo ejemplo, realizan la fotosíntesis). ¿Qué es lo que produciría este Libro quinto? ¿Qué efecto se seguiría de él de manera más inmediata, más reconocible?

De nuevo nos da una pista de ello su título, que en este caso es el siguiente: "Nosotros, los sin miedo». Extraño título, sin duda, pues en él no se nos dice a qué deberíamos temer, en qué consiste ese miedo que "nosotros" no tenemos. En este sentido puede resultar orientativo el aforismo 343, con el que comienza dicho libro. Otras épocas sintieron el temor de Dios, pero la nuestra, marcada decisivamente por el acontecimiento que conocemos bajo el nombre de "muerte de Dios", ¿qué otra cosa podría temer sino la "larga profusión y sucesión de derribo, destrucción, hundimiento, derrumbe que nos espera" (Nietzsche, 2014: 858) por haber sido "nosotros" ("vosotros y yo"; véase el aforismo 125) quienes hemos matado a Dios? Hasta el momento, prosigue Nietzsche, sólo hemos experimentado, sin embargo, libertad como resultado de este crimen; el peligro nos resulta estimulante y sentimos que otra vez nos están de nuevo permitidas "todas las empresas arriesgadas del hombre de conocimiento" (Nietzsche, 2014: 859).

Pero si, con todo, somos todavía en alguna medida piadosos (como sugiere el título del aforismo 344) es debido a que, aunque la creencia en Dios ya no articule nuestras vidas, mientras sigamos creyendo en valores absolutos no habremos hecho otra cosa que sustituir la idea de Dios por otras ideas igualmente "divinas", manteniendo intacto por ello el lugar esta idea ocupaba. La indagación acerca de la ciencia alegre se prolonga, así, en el estudio de 
la relación que dicha disciplina mantiene con la moral, de manera que sólo podremos denunciar la sustitución de Dios por la verdad (es decir, por la vieja concepción de la ciencia, que la mantiene por principio al margen de toda alegría), si somos capaces de romper con la interpretación de la verdad que hace de ella algo intrínsecamente "bueno" (esto es, con el sentido moral de la verdad). No tenemos temor, pero aún no nos hemos desligado del todo de la condición general que permitió a la humanidad sentir temor en otro tiempo. Es para alcanzar esta suprema libertad (y no con vistas al fomento de ninguna nostalgia religiosa) que debemos investigar el origen de nuestros prejuicios morales, tema que ya había sido analizado por Nietzsche en Aurora y que este autor retomará después de Asi habló Zaratustra, en una trayectoria que le conducirá hasta La genealogía de la moral.

\section{Bibliografía}

Cano, Germán, 2001, "Soy una máquina que puede estallar", en Friedrich Nietzsche, La ciencia jovial [La gaya scienza], Germán Cano (intro., trad., y notas), Madrid, Biblioteca Nueva, Biblioteca nietzscheana, pp. 11-49.

Colli, Giorgio, 2000, Introducción a Nietzsche, Romeo Medina (trad.), Valencia, Pre-Textos.

Foucault, Michel, 2000, La verdad y las formas jurídicas, Enrique Lynch (trad.), Barcelona, Gedisa.

Montinari, Mazzino, 1999, Lo que dijo Nietzsche, Enrique Lynch (trad.), Barcelona, Salamandra.

Nietzsche, Friedrich, 2008, Fragmentos póstumos, volumen II (1875-1882), Manuel Barrios y Jaime Aspiunza (intro., trad., y notas), Madrid, Tecnos. 
, 2010, Correspondencia, volumen IV, enero 1880-diciembre 1884, Marco Parmeggiani (intro., trad., apéndice y notas), Madrid, Tecnos.

, 2014,Obras completas, volumen III, escritos de madurez I, Jaime Aspiunza, Marco Parmeggiani, Diego Sánchez Meca y Juan Luis Vermal (intro., trad., y notas), Madrid, Tecnos.

Spinoza, Baruj, 2000, Ética demostrada según el orden geométrico, Atilano Rodríguez (trad., ed.), Madrid, Trotta.

Recibido: 5 de mayo de 2016 Aceptado: 11 de noviembre de 2016 